\title{
Set-membership Identification and Fault Detection using a Bayesian Framework
}

\author{
Rosa M. Fernández-Cantí, Joaquim Blesa, Vicenç Puig and Sebastian Tornil-Sin
}

Advanced Control Systems Group (SAC)

Universitat Politècnica de Catalunya (UPC), Pau Gargallo 5, 08028 Barcelona, Spain

\author{
Institut de Robòtica i
}

Informàtica Industrial, CSIC-UPC, Llorens i Artigas, Barcelona, Spain

e-mail: rfernandez@tsc.upc.edu, \{joaquim.blesa,vicenc.puig,sebastian.tornil\}@upc.edu

\begin{abstract}
This paper deals with the problem of set-membership identification and fault detection using a Bayesian framework. The paper presents how the setmembership model estimation problem can be reformulated from a Bayesian viewpoint in order to, firstly, determine the feasible parameter set in the identification stage and, secondly, check the consistency between the measurement data and the model in the fault detection stage. The paper shows that, assuming uniform distributed measurement noise and uniform model prior probability distributions, the Bayesian approach leads to the same feasible parameter set than the well-known set-membership technique based on approximating the feasible parameter set using sets. Additionally, it can deal with models that are nonlinear in the parameters. The single output and multiple output cases are addressed as well. The procedure and results are illustrated by means of the application to a quadruple tank process.
\end{abstract}

Keywords - Set-membership identification, fault detection, likelihood function, Bayes rule

\section{Introduction}

In the Control Engineering field, the so-called Robust Identification techniques deal 
with the problem of obtaining not only a nominal model of the plant, but also an estimate of the uncertainty associated to the nominal model. Such model of uncertainty is typically characterized as a region in the parameter space or as an uncertainty band around the frequency response of the nominal model.

Uncertainty models have been widely used in the design of robust controllers (Sánchez Peña and Sznaier, 1998) and, recently, their use in model-based fault detection procedures is increasing (Chen and Patton, 1999; Reppa and Tzes, 2011). In this later case, consistency between the new measurements and the parameter uncertainty region is checked. When an inconsistency is found, the existence of a fault is decided.

There exist two main approaches to characterize model uncertainty: the deterministic/worst case methods and the stochastic/probabilistic methods. For a survey, see e.g. (Reinelt, Garulli, and Ljung, 2002). Deterministic methods lead to hard bounds on the uncertainty region and the most representative are the set membership (SM) techniques (Milanese and Taragna, 2002; Milanese and Taragna, 2005) and the deterministic versions of the model error modelling (MEM) approach (Garulli and Reinelt, 2000).

Stochastic methods such as the Non Stationary Stochastic Embedding (NSSE) (Goodwin, Braslavsky, and Seron, 2002) lead to probabilistic bounds on the uncertainty region. In early years, probabilistic bounds were considered not suitable to describe uncertainty regions, but recent advances in robust risk adjusted controllers (Lagoa and Sznaier, 2005) and probabilistic fault detection (Jaulin, 2010) have given raise to stochastic methods. In particular, there is a renewed interest for the Bayesian point of view in system identification (Ninness and Henriksen, 2010; Schön, Wills, and Ninness, 2011). The topic is not new since early works in system identification already 
considered the Bayesian parameter estimation problem (Eykhoff, 1974) and model classification problem (Peterka, 1981). Moreover, the relationship between deterministic methods and stochastic methods was already explored in (Ninness and Goodwin, 1995) on the basis of Bayesian estimation. However, the Bayesian ideas, although appealing, have largely not been implemented due to the difficulty of computing the integrations involved in the posterior distributions. Recent advances in simulation techniques such as the Markov chain Monte Carlo (MCMC) have overcome this situation (Bolstad, 2010). In this sense, particle filtering is widely used in situations when state estimations are required (Arulampalam et al., 2002) and it is also applied in fault diagnosis problems (Verma et al., 2004). In this paper, we focus on the problem of set-membership parameter estimation for fault detection purposes, letting the extension to state estimation (as its relation with particle filters) as future research.

The aim of the paper is to present how the set-membership model estimation problem can be reformulated such that the Bayesian framework can be used to characterize the feasible parameter set (FPS) and to check the consistency between the measurement data and the model for fault detection purposes. Moreover, the proposed setmembership Bayesian approach is compared with the deterministic set-membership approach (Milanese, 1996) discussing the advantages and disadvantages of both approaches. The paper shows that the Bayesian approach, assuming uniform distributed measurement noise and uniform model prior probability distribution, leads to an inner approximation of the FPS differently from the outer approximation provided by the setmembership approach that uses sets to approximate the FPS. The motivation for using the Bayesian methodology to solve set-membership estimation problem is that it can deal with dynamic models that are nonlinear in the parameters. On the other hand, the resulting FPS obtained with fault free data can be used for fault detection purposes 
checking the consistency of the FPS with new data (Blesa, Puig, and Saludes, 2012). If there is an inconsistency with new data and the FPS, a fault is present in the system, and reinitializing the FPS to a large enough set, change in the healthy parameters can be determined as was proposed in (Ingimundarson et al, 2009).

This paper is organized as follows: Section 2 establishes the model parameterization that is going to be used and formulates the parameter set estimation problem and the fault detection problem. Section 3 addresses the two problems from a Bayesian viewpoint. In particular, we define the so-called Bayesian credible model set and particularize it in order to solve the set-membership parameter estimation problem. We also derive a test to check for faults on the basis of the resulting feasible parameter set. Section 4 illustrates the application of the proposed method to a quadruple tank process and presents the results in both the linear and the nonlinear cases. Finally, Section 5 concludes the paper.

\section{Problem Definition}

\subsection{Model parameterization}

Let us assume that the system can be expressed by means of the following regression model for

$$
y(k)=F(k, \boldsymbol{\theta})+e(k)=\hat{y}(k, \boldsymbol{\theta})+e(k), \quad k=1, \ldots, M
$$

where $F(k, \boldsymbol{\theta})$ is the regression (or observation) function, which in a general case is assumed to be nonlinear in the parameters $\boldsymbol{\theta}$, and it can contain any function of the 
system inputs $u(k)$ and outputs $y(k)$, where $k$ is the discrete time sample. The regression function can be viewed as the estimate of the system response produced by the model with parameters $\boldsymbol{\theta}, \hat{y}(k) \equiv F(k, \boldsymbol{\theta}) . \boldsymbol{\theta} \in \boldsymbol{\Theta}_{o}$ is the parameter vector of dimension $n_{\theta} \times 1 . \boldsymbol{\Theta}_{o}$ is the set in the parameter space whose boundary represents the $a$ priori bounds for the parameter values. $e(k)$ is an additive error term which is unknown but it is assumed to be bounded by a constant $|e(k)| \leq \sigma$.

Remark 1: Model parameterization (1) is introduced in order to better formalize the proposed Bayesian set-membership approach and to allow a comparison with set-based set-membership approaches (Milanese, 1996) that use the same type of parameterization.

\subsection{Parameter estimation problem}

Given a sequence of input and output data (rich enough from the identificability point of view and without no faults and outliers) collected from the system defined as

$$
\tilde{y}_{M}=\{y(k)\}_{k=1, \ldots, M}, \quad \tilde{u}_{M}=\{u(k)\}_{k=1, \ldots, M}
$$

the parameter estimation problem can be defined as follows:

Definition 1: Given the system input and output sequences $\left(\tilde{y}_{M}, \tilde{u}_{M}\right)$, the system model expressed in regressor form (1), the noise bound $\sigma$ and the initial parameter set $\boldsymbol{\Theta}_{o}$, the set-membership parameter estimation problem consists in determining the Feasible Parameter Set (FPS) defined as the set of parameters $\boldsymbol{\theta}$ such that satisfy

$$
|y(k)-F(k, \boldsymbol{\theta})| \leq \sigma, \quad k=1, \cdots, M, \quad \boldsymbol{\theta} \in \boldsymbol{\Theta}_{o}
$$

The FPS can be defined in compact form as follows 


$$
\mathrm{FPS}=\left\{\boldsymbol{\theta} \in \boldsymbol{\Theta}_{o} \mid y(k)-\sigma \leq F(k, \boldsymbol{\theta}) \leq y(k)+\sigma, k=1, \cdots, M\right\}
$$

In the case that the regression function is expressed linearly as $F(k, \boldsymbol{\theta})=\boldsymbol{\varphi}^{T}(k) \boldsymbol{\theta}$, the model parameterization (1) can be expressed as

$$
y(k)=\varphi^{T}(k) \boldsymbol{\theta}+e(k)=\hat{y}(k)+e(k)
$$

where $\varphi^{T}(k)$ is the regressor vector of dimension $1 \times n_{\theta}$. In this case, if the bound parameter set $\boldsymbol{\Theta}_{O}$ is described by a convex polytope that can be expressed in the $\mathcal{H}$ polytope form (Ziegler, 1995) as $\boldsymbol{\Theta}_{o}=\left\{\boldsymbol{\theta} \in \mathbb{R}^{n_{\theta}} \mid \mathbf{A}_{0} \boldsymbol{\theta} \leq \mathbf{b}_{0}\right\}$ with $\mathbf{A}_{0} \in \mathbb{R}^{n \times n_{\theta}}$ and $\mathbf{b}_{0} \in \mathbb{R}^{n}$ the FPS is also a convex polytope (Blesa, Puig, and Saludes, 2012) that can be described in the $\mathcal{H}$-polytope form as

$$
\operatorname{FPS}=\left\{\boldsymbol{\theta} \in \mathbb{R}^{n_{\theta}},\left(\begin{array}{c}
\mathbf{A}_{0} \\
\mathbf{A}
\end{array}\right) \boldsymbol{\theta} \leq\left(\begin{array}{c}
\mathbf{b}_{0} \\
\mathbf{b}
\end{array}\right)\right\}
$$

with

$$
\mathbf{A} \in \mathbb{R}^{2 M \times n_{\theta}}=\left(\begin{array}{c}
-\boldsymbol{\varphi}^{T}(1) \\
\boldsymbol{\varphi}^{T}(1) \\
\vdots \\
-\boldsymbol{\varphi}^{T}(M) \\
\boldsymbol{\varphi}^{T}(M)
\end{array}\right), \quad \mathbf{b} \in \mathbb{R}^{2 M}=\left(\begin{array}{c}
-y(1)+\sigma \\
y(1)+\sigma \\
\vdots \\
-y(M)+\sigma \\
y(M)+\sigma
\end{array}\right)
$$

Also, in the linear regression case, the FPS can be obtained by intersecting all the $M$ strips defined by the pairs of parallel lines $\left(y(k)-\varphi^{T}(k) \boldsymbol{\theta}=\sigma\right.$ and $y(k)-\varphi^{T}(k) \boldsymbol{\theta}=-\sigma$ separated by $2 \sigma$. In the case that the regression function $F(k, \theta)$ is nonlinear in the parameters $\boldsymbol{\theta}$, the resulting FPS is no longer a convex polytope but a region with a much more complicated shape.

In order to avoid dealing with the exact description of the FPS several algorithms exist 
that obtain inner or outer simpler regions that approximate the exact FPS. Such regions are known as Approximated Feasible Parameter Sets (AFPS).

Inner approximations find the approximate parameter set of maximum volume AFPS $_{i n}$ such that all its parameters are inside the feasible parameter set,

$$
\mathrm{AFPS}_{\text {in }} \subseteq \text { FPS } .
$$

On the other hand, outer approximation algorithms find the approximate parameter set of minimum volume AFPS $_{\text {out }}$ that guarantees that the feasible parameter set is inside it,

$$
\mathrm{FPS} \subseteq \mathrm{AFPS}_{\text {out }}
$$

When $F(k, \boldsymbol{\theta})$ is linear, boxes, parallelotopes, ellipsoids or zonotopes are used to characterize the AFPS (Alamo, Bravo, and Camacho, 2005; Blesa, Puig, and Saludes, 2011). In the nonlinear case, a minimum outer box can be determined by means of a set of optimization problems (Milanese et al., 1996). But since the parameters enter in a nonlinear way in (1), the resulting optimization problems are nonconvex.

As an alternative to nonconvex optimization, recursive algorithms can be used as follows

$$
\operatorname{FPS}(k)=\mathrm{FPS}(k-1) \cap \mathrm{S}(k)
$$

where $\mathrm{S}(k)$ is the set of parameters consistent with data at the sample $k$

$$
\mathrm{S}(k)=\left\{\boldsymbol{\theta} \in \mathbb{R}^{n_{\theta}} \mid-\sigma \leq y(k)-F(k, \boldsymbol{\theta}) \leq \sigma\right\}
$$

Recursive algorithms allow the efficient computation of inner

$$
\operatorname{AFPS}_{i n}(k) \subseteq \operatorname{AFPS}_{i n}(k-1) \cap \mathrm{S}(k)
$$


or outer approximations

$$
\mathrm{AFPS}_{\text {out }}(k-1) \cap \mathrm{S}(k) \subseteq \mathrm{AFPS}_{\text {out }}(k)
$$

In this latter approach, the AFPS can be estimated by using subpavings and the SIVIA (Set Inversion Via Interval Analysis) algorithm which is based on refining the initial $a$ priori set $\boldsymbol{\Theta}_{o}$ by iteratively bisecting it (Jaulin, Kieffer, Didrit, and Walter, 2001).

\subsection{Fault detection in the set-membership framework}

Once the FPS (or its approximation) has been estimated with nonfaulty data, it can be used for fault detection.

The fault detection problem in the set-membership can be defined as follows:

Definition 2: Given the system input and output sequences $\left(\tilde{y}_{M}, \tilde{u}_{M}\right)$, the system model expressed in regressor form (1), the noise bound $\sigma$ and the initial parameter set $\boldsymbol{\Theta}_{o}$, the set-membership fault detection problem consists in determining if

$$
\mathrm{FPS}=\left\{\boldsymbol{\theta} \in \boldsymbol{\Theta}_{o} \mid y(k)-\sigma \leq F(k, \boldsymbol{\theta}) \leq y(k)+\sigma, k=1, \cdots, M\right\}=\varnothing
$$

to prove the inconsistency between the input/output data and the model. In the inconsistency is proved, a fault is indicated.

The fault detection test can be implemented in a recursive way by checking if the set of parameters consistent with data at the sample $k(9)$ is inconsistent with the FPS. The inconsistency can be checked by means of the intersection of $\mathrm{S}(k)$ with the FPS. A fault will be indicated if this intersection leads to an empty set

$$
\mathrm{S}(k) \cap \mathrm{FPS}=\varnothing
$$

In the linear case, if the identification data length is moderate, the fault detection test 
(12) can be solved efficiently by determining the feasibility of a linear optimization problem.

\subsection{Extension to the multiple output case}

The previous results can be extended to the case of multiple outputs. Consider now that system to be monitored can be expressed by means of the following multiple output regression model

$$
\mathbf{y}(k)=\mathbf{F}(k, \boldsymbol{\theta})+\mathbf{e}(k)=\hat{\mathbf{y}}(k, \boldsymbol{\theta})+\mathbf{e}(k), \quad k=1, \ldots, M
$$

where the vector regression function $\mathbf{F}(k, \boldsymbol{\theta})$ can be nonlinear in the parameters $\boldsymbol{\theta}$, and it contains any function of vector inputs $\mathbf{u}(k)$ and outputs $\mathbf{y}(k)$ and can be decomposed in $n_{y}$ components $\mathbf{F}(k, \boldsymbol{\theta})=\left(F_{1}(k, \boldsymbol{\theta}), \cdots, F_{n_{y}}(k, \boldsymbol{\theta})\right)^{T}$ in such a way that

$$
\begin{gathered}
y_{1}(k)=F_{1}(k, \boldsymbol{\theta})+e_{1}(k)=\hat{y}_{1}(k, \boldsymbol{\theta})+e_{1}(k) \\
\vdots \\
y_{n_{y}}(k)=F_{n_{y}}(k, \boldsymbol{\theta})+e_{n_{y}}(k)=\hat{y}_{n_{y}}(k, \boldsymbol{\theta})+e_{n_{y}}(k)
\end{gathered}
$$

where $y_{1}(k), \cdots, y_{n y}(k)$ are the $n_{y}$ components of the output vector $\mathbf{y}(k)$ and $e_{1}(k), \cdots, e_{n y}(k)$ are the $n_{y}$ components of the additive error vector $\mathbf{e}(k)$ which is unknown but it is assumed to be bounded by a constant $\left|e_{i}(k)\right| \leq \sigma_{i}, \forall k, \quad i=1, \cdots, n_{y}$.

Now, the parameter set consistent to input/output data at sample $k, \mathrm{~S}(k)$, is defined as the intersection of the consistency sets corresponding to each output $i, \mathrm{~S}_{i}(k), i=1, \ldots, n_{y}$, at the same sample $k$

$$
\mathrm{S}(k)=\bigcap_{i=1}^{n_{y}} \mathrm{~S}_{i}(k)
$$

with 


$$
\mathrm{S}_{i}(k)=\left\{\boldsymbol{\theta} \in \mathbb{R}^{n_{\theta}} \mid-\sigma_{i} \leq y_{i}(k)-F_{i}(k, \boldsymbol{\theta}) \leq \sigma_{i}\right\}
$$

The set $\mathrm{S}(k)$ can be expressed in a compact form as follows

$$
\mathrm{S}(k)=\left\{\begin{array}{l|l}
\boldsymbol{\theta} \in \mathbb{R}^{n_{\theta}} & \begin{array}{c}
-\sigma_{1} \leq y_{1}(k)-F_{1}(k, \boldsymbol{\theta}) \leq \sigma_{1} \\
\vdots \\
-\sigma_{n_{y}} \leq y_{n_{y}}(k)-F_{n_{y}}(k, \boldsymbol{\theta}) \leq \sigma_{n_{y}}
\end{array}
\end{array}\right\}
$$

In the same way, the FPS corresponding to the multiple output case is now

$$
\mathrm{FPS}=\left\{\begin{array}{l|l}
\boldsymbol{\theta} \in \Theta_{0} & \begin{array}{c}
-\sigma_{1} \leq y_{1}(k)-F_{1}(k, \boldsymbol{\theta}) \leq \sigma_{1} \\
\vdots \\
-\sigma_{n_{y}} \leq y_{n_{y}}(k)-F_{n_{y}}(k, \boldsymbol{\theta}) \leq \sigma_{n_{y}}
\end{array} \quad, \forall k=1, \ldots, M
\end{array}\right\}
$$

As in the single output case, if the regression function $\mathbf{F}(k, \boldsymbol{\theta})$ is linear in the parameters

$\boldsymbol{\theta}$, the multiple output FPS can be described as a convex polytope. Thus, the setmembership estimation and fault detection procedures described in the previous sections for the single output case can be used for the multiple output case as well.

\section{Set-membership Estimation and Fault Detection in the Bayesian Framework}

Once the set-membership parameter estimation and fault detection problems have introduced, an approach to solve them based on the Bayesian framework is proposed.

\subsection{Bayesian set-membership parameter estimation}

The parametric-type uncertainty can be described by means of the Bayesian credible parameter set:

$$
\mathscr{B}_{\boldsymbol{\theta}} \equiv\left\{\boldsymbol{\theta} \in \boldsymbol{\Theta}_{0}: p(\boldsymbol{\theta} \mid \mathbf{y}) \geq c(\alpha)\right\}
$$


where the process model is characterized by means of the parameter vector $\boldsymbol{\theta}$, $\mathbf{y}=\left(\begin{array}{lll}y(1) & \ldots & y(M)\end{array}\right)^{T}$ is the measurement data vector, $c(\alpha)$ is the critical value where $100(1-\alpha) \%$ is the desired credibility level, and the model posterior distribution can be obtained by means of the Bayes' rule,

$$
p(\boldsymbol{\theta} \mid \mathbf{y})=\frac{p(\mathbf{y} \mid \boldsymbol{\theta}, \sigma) p(\boldsymbol{\theta})}{p(\mathbf{y})}
$$

where $p(\mathbf{y} \mid \boldsymbol{\theta}, \sigma)$ is the likelihood of the observations $\mathbf{y}$ jointly conditioned to the model $\boldsymbol{\theta}$ and to the error bound $\sigma, p(\boldsymbol{\theta})$ is the prior distribution on the model parameters, and $p(\mathbf{y})$ is just a normalized constant, so we can express the posterior as $p(\boldsymbol{\theta} \mid \mathbf{y}) \propto p(\mathbf{y} \mid \boldsymbol{\theta}, \sigma) p(\boldsymbol{\theta})$.

Note that although the Bayesian credible parameter set (19) is a very general set suitable for most identification procedures, including nonparametric and nonstandard distributions, it is not necessary to use it in its full powerfulness for the parameter set estimation problem considered here. A simpler version, obtained by taking the assumptions that are listed below, will be enough.However, different assumptions would lead to different types of AFPSs, some of them, characterized by more complete descriptions of the posterior distribution, and surely enjoying different and interesting properties, but for the sake of clearness here we focus on the simplest case.

Hence, the following assumptions allow showing that the FPS defined in Section 2 can be approximated from a Bayesian approach. These assumptions lead to a point-wise inner approximation of (2).

The assumptions taken in this work are the following: 
First assumption: The prior distribution is uniform. In the Bayesian framework, the model prior probability distribution $p(\boldsymbol{\theta})$ can be a subjective probability (Robert, 2001). For simplicity, here it is assumed that no information about which the value of the "true" parameter vector $\boldsymbol{\theta}$ will be and consequently we take a uniform $p(\boldsymbol{\theta})$ which is flat over the initial set $\boldsymbol{\Theta}_{o}$. This way the model posterior distribution is directly proportional to the likelihood function of the observations, $p(\boldsymbol{\theta} \mid \mathbf{y}) \propto p(\mathbf{y} \mid \boldsymbol{\theta}, \sigma)$ in the considered initial support $\boldsymbol{\Theta}_{o}$.

Since for a fixed $\boldsymbol{\theta}, \hat{y}(k)=F(k, \boldsymbol{\theta}), \forall k$, are deterministic quantities, the likelihood $p(\mathbf{y} \mid \boldsymbol{\theta}, \sigma)$ coincides in form with the error term distribution, i.e., $p(\mathbf{y} \mid \boldsymbol{\theta}, \sigma) \equiv p_{e}(\mathbf{y}-\hat{\mathbf{y}} \mid \sigma)$, where $\hat{\mathbf{y}}=\left(\begin{array}{lll}\hat{y}(1) & \ldots & \hat{y}(M)\end{array}\right)^{T}$.

Second assumption: The error term is uniform distributed. To obtain a hard-bounded uncertainty region (credible region in the Bayesian terminology), we must assume that the distribution of the additive error is hard-bounded. The simplest choice is to take the uniform distribution, i.e., $e(k) \sim \mathcal{U}(-\sigma, \sigma), \forall k$, where $\sigma$ is selected to be the additive error bound presented in Section 2. In this case, the resulting likelihood function is constant and nonzero in the region where models $\boldsymbol{\theta}$ are consistent with the measurements and it is zero outside this region.

Note that, unlike particle filtering approaches (Arulampalam et al., 2002), we are not really concerned on obtaining the posterior distribution for $\boldsymbol{\theta}$; instead, what we obtain is the region (within the initial support $\boldsymbol{\Theta}_{o}$ ) for which the posterior distribution for $\boldsymbol{\theta}$ is constant and nonzero. This region will serve as an inner characterization of the AFPS. Note also that, since the value of the posterior distribution for $\boldsymbol{\theta}$ is constant over the 
FPS, the $\alpha$ value is not relevant here either. All models $\boldsymbol{\theta}$ will be equally probable to occur, and the probability level will be related to the FPS size. If we were interested in different levels of probability inside the hard-bounded FPS we could use different prior distributions for $\boldsymbol{\theta}$, e.g. Gaussian distributions. Still, if we were interested in softbounded FPSs we could use soft-bounded likelihood functions instead of uniform likelihood functions. These two later situations are out of the scope of this paper and will not be considered here.

Third assumption: Equation-error assumption. The likelihood function can be numerically estimated by taking the so-called equation-error assumption. On the contrary to the error-in-variables approach, where the regression function itself presents an error term, the equation-error approach assumes that the error term is additive to the data at each time sample $k$. This assumption was early justified in (Sorenson, 1970) and, since it significantly simplifies the procedures, it is assumed in most set-membership parametric techniques (Milanese et al., 1996).

This way, we can assume that the error samples $e(k)=y(k)-\hat{y}(k), \forall k$ are i.i.d. (independent and identically distributed) and we can compute the likelihood function numerically and sample-to-sample,

$$
p_{e}(\mathbf{y}-\hat{\mathbf{y}} \mid \boldsymbol{\theta}, \sigma)=\prod_{k=1}^{M} p_{e}(y(k)-\hat{y}(k) \mid \boldsymbol{\theta}, \sigma)
$$

Note that there is no difference in the computation of $(21)$ whether $F(k, \boldsymbol{\theta})$ is linear in the parameters or not. The likelihood function (21) can be numerically estimated by using a Monte Carlo approach, see e.g. (Ninness and Henriksen, 2010; Schön et al., 2010). This is the choice in most Bayesian works. However, in the next section, we estimate it by means of the gridding of the candidate parameter vectors $\boldsymbol{\theta}_{i}$ and taking 
the equation-error assumption.

\subsection{Computation of the point-wise Bayesian AFPS}

The approximation for the FPS in the Bayesian approach is the following:

$$
\mathrm{AFPS}_{\mathcal{B}}=\left\{\boldsymbol{\theta}_{i} \in \boldsymbol{\Theta}_{o} \mid p_{e}\left(y(k)-F\left(k, \boldsymbol{\theta}_{i}\right) \mid \boldsymbol{\theta}_{i}, \sigma\right) \neq 0, k=1, \cdots, M, i=1, \ldots, N\right\}
$$

The simplest procedure to compute (22) consists in taking an arbitrary point grid in $\boldsymbol{\Theta}_{o}$ defined by a pre-specified distance between points. Each point $\boldsymbol{\theta}_{i}$ corresponds to a possible model for the physical plant. Then, for each time sample and model $\boldsymbol{\theta}_{i}$, we compute the error between the measurement and the predicted output $\hat{y}(k)=F\left(k, \boldsymbol{\theta}_{i}\right)$. If this error is inside the bound, we conclude that the model is consistent with the measurements and we assign it a likelihood value of one, $p_{e}\left(y(k)-\hat{y}(k) \mid \boldsymbol{\theta}_{i}, \sigma\right)=1$; otherwise, the likelihood is zero. This procedure is repeated for all $M$ samples for all the $N$ points $\boldsymbol{\theta}_{i}$ in the grid.

A high level description of the procedure is summarized in Algorithm 1. 
Algorithm 1 Set-membership identification using point-based evaluation

$\{\boldsymbol{\theta}\} \leftarrow$ create_grid $\left(\Theta_{0}, \varepsilon\right)$

$k \leftarrow 1$

while $(k \leq M)$ do

$\theta_{i} \leftarrow$ obtain_first $(\{\theta\})$

while $\left(\theta_{i} \neq \varnothing\right)$ do

$\hat{y} \leftarrow F\left(k, \theta_{i}\right)$

$e \leftarrow y(k)-\hat{y}$

if $|e|>\sigma$ then

$\operatorname{delete}\left(\{\theta\}, \theta_{i}\right)$

end if

$\theta_{i} \leftarrow$ obtain_next $(\{\theta\})$

end while

$k \leftarrow k+1$

end while

$\operatorname{return}(\{\theta\})$

end algorithm

Algorithm 1 uses a list of points $\{\boldsymbol{\theta}\}$, i.e. a list of combinations of values for the parameters. At the beginning of the algorithm, the list is initialized with the grid of points selected inside the initial set $\Theta_{0}$. Through two nested while loops, the algorithm applies the available data to the elements in the list, deleting the combinations of 
parameters that are proven to be inconsistent with the data at any time instant. The algorithm returns a list of points that belong to the FPS, hence providing an inner AFPS.

Remark 2: Since all the $M$ observations in Algorithm 1 should be included in the output prediction zonotope, the presence of exceptional data points (i.e., outliers) may lead to empty or marginal FPS. In order to address this issue, algorithms to remove outliers (like the ones proposed in Campi. Calafiore and Garatti, 2009) should be applied such that $M_{o}<M$ data are discarded and apply the remaining $M-M_{o}$ data to Alogrithm 1 .

\subsection{Fault detection in the Bayesian framework}

Once we have calibrated the Bayesian model (i.e., we have obtained the samples of the likelihood function $p_{e}\left(\mathbf{y}-\hat{\mathbf{y}} \mid \boldsymbol{\theta}_{i}, \sigma\right)$ for all the points $\boldsymbol{\theta}_{i}$ in the parameter grid), the detection of faults can be carried out for every new measurement $y(k), k \geq M+1$, by computing the new likelihood function $p_{e}\left(y(k)-\hat{y}(k) \mid \boldsymbol{\theta}_{i}, \sigma\right), \forall \boldsymbol{\theta}_{i}$, and verifying whether there is at least one parameter vector $\boldsymbol{\theta}_{j}$ in the grid for which both the calibrated likelihood $p_{e}\left(\mathbf{y}-\hat{\mathbf{y}} \mid \boldsymbol{\theta}_{j}, \sigma\right)$ and the new likelihood $p_{e}\left(y(k)-\hat{y}(k) \mid \boldsymbol{\theta}_{j}, \sigma\right), k \geq+1$, are nonzero. If this parameter (or set of parameters) exists, we conclude that the new measurement is consistent with the AFPS.

The consistency can be checked by simply multiplying both likelihood functions for each parameter $\boldsymbol{\theta}_{i}$ in the grid. If the product is equal to zero for all the parameters in the grid,

$$
p_{e}\left(\mathbf{y}-\hat{\mathbf{y}} \mid \boldsymbol{\theta}_{i}, \sigma\right) \times p_{e}\left(y(k)-\hat{y}(k) \mid \boldsymbol{\theta}_{i}, \sigma\right)=0, \forall \boldsymbol{\theta}_{i}, k \geq M+1
$$

we decide that a fault has taken place. Since we consider a sample at once, the test (23) 
can be implemented on-line by means of Algorithm 2 .

Algorithm 2 Set-membership fault detection using point-based evaluation $\{\boldsymbol{\theta}\} \leftarrow \mathrm{AFPS}_{\mathcal{B}}$

$k \leftarrow 1$

$F D \leftarrow 0$

while $(k \leq$ end $)$ do

$\theta_{i} \leftarrow$ obtain_first $(\{\theta\})$

while $\left(\theta_{i} \neq \varnothing\right)$ do

$$
\begin{aligned}
& \hat{y} \leftarrow F\left(k, \theta_{i}\right) \\
& e \leftarrow y(k)-\hat{y} \\
& \text { if }|e|>\sigma \text { then } \\
& \quad \operatorname{delete}\left(\{\theta\}, \theta_{i}\right)
\end{aligned}
$$

end if

$$
\boldsymbol{\theta}_{i} \leftarrow \text { obtain_next }(\{\boldsymbol{\theta}\})
$$

end while

if (is_empty $(\{\boldsymbol{\theta}\}))$ then

$$
\begin{aligned}
& F D \leftarrow 1 \\
& \{\theta\} \leftarrow \text { create_grid }\left(\Theta_{0}, \varepsilon\right)
\end{aligned}
$$

end if

$k \leftarrow k+1$

end while

end algorithm 
Algorithm 2 for fault detection is a modification of Algorithm 1 for parameter estimation. However, it must be noticed that both algorithms will be used under different conditions: Algorithm 1 will be applied off-line using data collected for system normal (fault-free) operation, while Algorithm 2 will be applied on-line with the goal of determining the system condition in real-time. The differences with Algorithm 1 are the following. First, in Algorithm 2 the initialization of the list of points in the parameter space $\{\boldsymbol{\theta}\}$ uses the results previously obtained by Algorithm 1. Second, if at any time instant $k$ the list of points becomes empty because none of the combinations of parameters in the list is consistent with the data, then the fault indicator FD is set and the list is reinitialized by using the a-priori initial parameter set $\Theta_{0}$. This allows the algorithm not only to detect faults but also to identify the magnitude for parametric faults.

Of course, the ability to detect "small" faults depends on the grid density (the number $N$ of candidate parameter vectors $\left.\boldsymbol{\theta}_{i}\right)$ but it does not depend on $c(\alpha)$ in the hardbounded case considered here (since all the models inside the AFPS are equally probable and the $\alpha$ value would only assign the percentage of probability corresponding to each of them). A denser grid would be able to detect smaller deviations of the parameter vector, because more uncertain nonfaulty models would be checked in (23) and thus we would have more models able to explain the normal behavior. Moreover, a denser grid would also decrease the number of false alarms, since the borders of the AFPS would contain more models able to explain the normal behavior. Although a denser grid implies a more intense calibration stage, it does not increase significantly the computation load in the fault detection stage. 


\subsection{Extension to the multiple output case}

The uncertainty calibration procedure explained in the previous section can be extended to the case of multiple output systems by taking the joint likelihood function of the $n_{y}$ outputs

$$
p_{e}\left(\mathbf{y}_{1}-\hat{\mathbf{y}}_{1}, \ldots, \mathbf{y}_{n_{y}}-\hat{\mathbf{y}}_{n_{y}} \mid \boldsymbol{\theta}, \sigma\right)=\prod_{k=1}^{M} p_{e}\left(y_{1}(k)-\hat{y}_{1}(k) \mid \boldsymbol{\theta}, \sigma\right) \times \cdots \times p_{e}\left(y_{n_{y}}(k)-\hat{y}_{n_{y}}(k) \mid \boldsymbol{\theta}, \sigma\right)
$$

And the fault detection procedure can be generalized in an analogous way,

$$
\begin{array}{r}
p_{e}\left(\mathbf{y}_{1}-\hat{\mathbf{y}}_{1}, \ldots, \mathbf{y}_{n_{y}}-\hat{\mathbf{y}}_{n_{y}} \mid \boldsymbol{\theta}_{i}, \sigma\right) \times p_{e}\left(y_{1}(k)-\hat{y}_{1}(k) \mid \boldsymbol{\theta}_{i}, \sigma\right) \times \cdots \times p_{e}\left(y_{n_{y}}(k)-\hat{y}_{n_{y}}(k) \mid \boldsymbol{\theta}_{i}, \sigma\right)=0, \\
\forall \boldsymbol{\theta}_{i}, k \geq M+1
\end{array}
$$

\subsection{Discussion}

The mainstream in set-membership parameter estimation considers the use of set-based methods. Typically, the result of a parameter estimation problem is a set of a given type that provides an outer approximation (as accurate as possible) of the exact FPS. A rich variety of approximating sets are proposed in the literature, e.g. boxes, ellipsoids, polytopes, zonotopes and subpavings. Unfortunately, some of the previous types of sets can only be used for the identification of linear systems. Boxes and subpavings, manipulated by using interval analysis methods, are the only ones that can be used for the identification of non-linear systems. And since boxes provide too rough approximations for arbitrary shaped sets, subpavings are at the end the only alternative. The standard set-based solution to the non-linear parameter estimation problem is the use of subpavings and the SIVIA (Set Inversion Via Interval Analysis) algorithm (Jaulin et al., 2001). Subpavings are unions of non-overlapping boxes that can approximate compact sets with arbitrary precision. The SIVIA algorithm provides an (outer) approximation of the subset of points in the domain whose evaluation by a given 
function lies in a prespecified image set. The SIVIA algorithm can be directly applied to the Set Membership parameter estimation problem, being the function to evaluate the regression function $F(k, \boldsymbol{\theta})$ in (1) and being the image set the box given by the addition of the uncertainty $\sigma$ to the measurements, i.e. $[y(k)]=[y(k)-\sigma, y(k)+\sigma], k=0, \cdots, M$. The proposed Bayesian Set Membership parameter estimation algorithm can be compared with SIVIA. The first aspect to consider is that SIVIA provides outer approximations of the exact FPS while the Bayesian method will provide inner approximations. Applied to the fault detection problem, this means that SIVIA will assure the elimination of false alarms but with a loss of sensitivity to faults. On the other hand, the use of the Bayesian method will lead to a given false alarm rate different from zero but without a loss of fault sensitivity (in fact, the sensitivity to faults will increase). To decide if it is worse to loose fault sensitivity or to have false alarms may depend on the application, but in general it depends on their magnitudes. And these magnitudes are associated to the quality of the outer and inner approximations of the FPS provided by the two methods. Both methods share the property of being able to provide approximations of arbitrary precision at a cost of computation time, but their performances can be compared working at fixed resolution levels. Some experiments using an example and detailed in the previous work (Fernández-Canti et al., 2013) suggest that for a given resolution level the quality of the inner approximation provided by the Bayesian method is expected to be higher than the quality of the outer approximation provided by SIVIA (excess of overbounding due to the well known multi-incidence problem of interval arithmetic). On the other hand, the computation time needed by the Bayesian method is expected to be lower than the needed by SIVIA. 


\section{Example}

A quadruple-tank process, proposed by (Johansson, 2000), is used to illustrate the procedures presented in this paper. The schematic diagram of the system is shown in Figure 1. The process inputs are the input voltages to the pumps, $v_{1}$ and $v_{2}$, and the process outputs are the tank levels $h_{i}, i=1, \cdots, 4$.

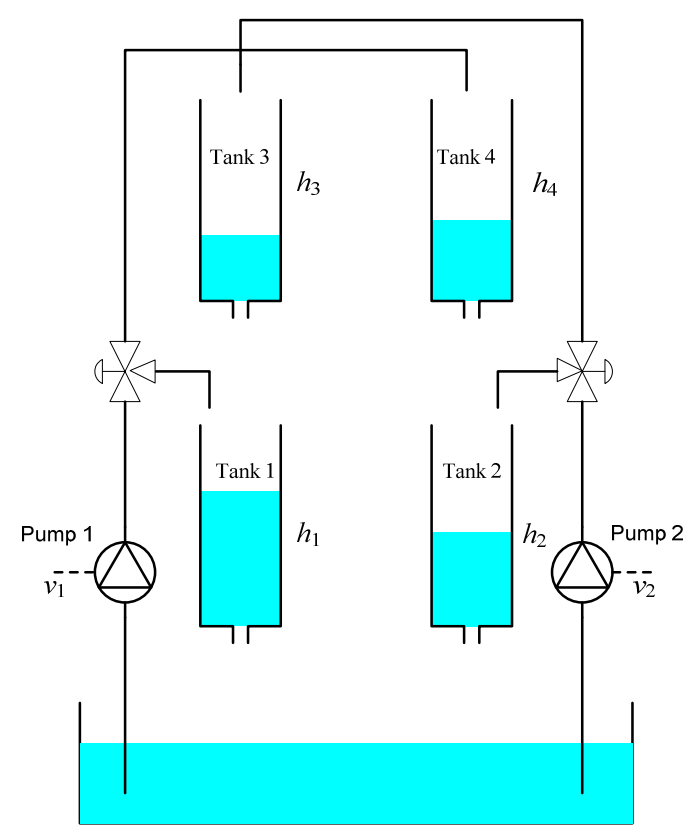

Figure 1. Quadruple-tank process

\subsection{Multi input single output model}

We firstly focus on a part of the whole system. We assume that the levels $h_{1}, h_{3}$ and the voltage $v_{1}$ can be directly measured. The equation that describes the dynamic behavior of this part of the system (output: $h_{1}$, inputs: $h_{3}, v_{1}$ ) is:

$$
\dot{h_{1}}=-\frac{a_{1}}{A_{1}} \sqrt{2 g h_{1}}+\frac{a_{3}}{A_{1}} \sqrt{2 g h_{3}}+\frac{\rho_{1} k_{1}}{A_{1}} v_{1}
$$

where $\dot{h}_{1}=d h_{1} / d t, a_{1}$ and $a_{3}$ are the cross-sections of the outlet holes of tanks 1 and 3 , and $A_{1}=28 \mathrm{~cm}^{2}$ is the cross-section of tank 1 . The term $k_{1} v_{1}$ with $k_{1}=3.33 \mathrm{~cm}^{3} / \mathrm{Vs}$ is the first 
pump flow and the parameter $\rho_{1}=0.7$ is determined from how the first valve is set prior to the experiment. The gravity acceleration is $g=981 \mathrm{~cm} / \mathrm{s}^{2}$. Finally, we consider that the operating range is $h_{1} \in[2,11] \mathrm{cm}$ and $h_{3} \in[1,15] \mathrm{cm}$.

The parameters $a_{1}$ and $a_{3}$ are the ones to be estimated and their nominal values are assumed to be $a_{1}=a_{3}=0.071 \mathrm{~cm}^{2}$.

\subsection{Discrete models}

Discrete models for the linear and nonlinear regression cases will be used to illustrate that the proposed approach works well in either case:

\subsubsection{Linear case}

A discrete, linearized version of (26) can be obtained by means of the forward approximation of the derivative $\dot{h}_{1} \approx\left(h_{1}(k)-h_{1}(k-1)\right) / T_{s}$ with sampling time $T_{s}=1 s$. This way, (26) can be expressed in the following linear regression form

$$
h_{1}(k)=h_{1}(k-1)+\varphi^{T}(k) \boldsymbol{\theta}+\frac{\rho k_{1}}{A_{1}} v_{1}(k-1)+e(k)
$$

where $\varphi^{T}(k)=\left(-\frac{1}{A_{1}} \sqrt{2 g h_{1}(k-1)} \frac{1}{A_{1}} \sqrt{2 g h_{3}(k-1)}\right)$ is the regressor vector and $\boldsymbol{\theta}=\left(\begin{array}{ll}a_{1} & a_{3}\end{array}\right)^{T}$ is the model parameter vector to be estimated. The term $e(k)$ is the additive error due to the measurement noise and discretization and it is assumed to be bounded, $|e(k)| \leq \sigma=0.05 \mathrm{~cm}$.

\subsubsection{Nonlinear case}


A model nonlinear in the parameters can be obtained if an output observer is used (see Figure 2).

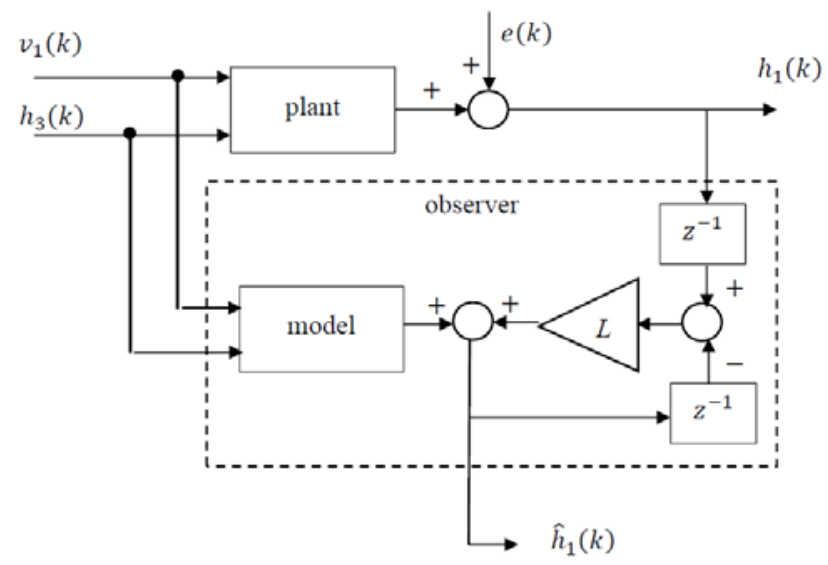

Figure 2. Multi Input Single Output plant with output observer

Observers improve the ability of detecting output faults but lead to structures nonlinear in the parameters. In our example, the resulting expression is

$$
\hat{h}_{1}(k)=\hat{h}_{1}(k-1)+\varphi^{T}(k) \boldsymbol{\theta}+\frac{\rho k_{1}}{A_{1}} v_{1}(k-1)+e(k)+L\left(h_{1}(k-1)-\hat{h}_{1}(k-1)\right)
$$

where $\varphi^{T}(k)=\left(-\frac{1}{A_{1}} \sqrt{2 g \hat{h}_{1}(k-1)} \frac{1}{A_{1}} \sqrt{2 g h_{3}(k-1)}\right), \boldsymbol{\theta}=\left(\begin{array}{ll}a_{1} & a_{3}\end{array}\right)^{T}$, and $|e(k)| \leq \sigma=0.05 \mathrm{~cm}$.

\subsection{Uncertainty estimation in a fault-free scenario}

To obtain the uncertainty region (FPS), i.e., to determine the uncertainty region for $a_{1}$ and $a_{3}$ in the parameter space, a set of $M=140$ measurements has been obtained in a fault-free scenario (see Figure 3). 

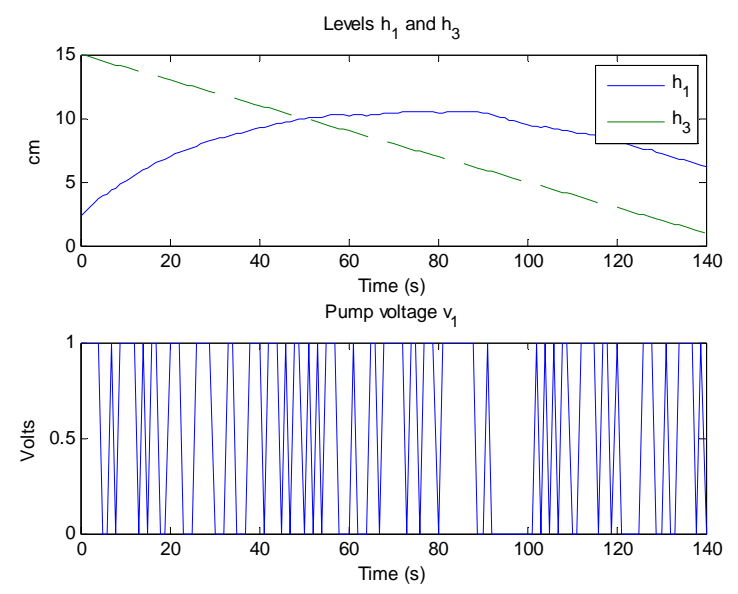

Figure 3. Identification scenario

\subsubsection{Linear case}

Figure 4 shows the FPS obtained by the strips intersection set-membership technique described in Section 2. The red little circles indicate the final (i.e., after $M$ intersections) polytope vertices.

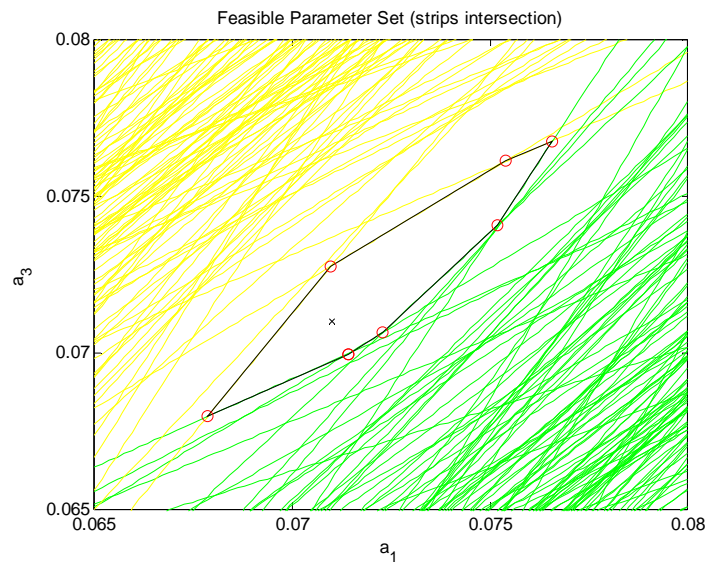

Figure 4. FPS obtained by the strips intersection technique

Figure 5 shows the FPS region obtained by computing the contour of the likelihood function assuming that the error is uniform distributed as $v(-\sigma, \sigma)$ for a grid of $60 \times 60$ parameters. As expected, this region coincides to the one obtained by the strips intersection method shown in Figure 4. 


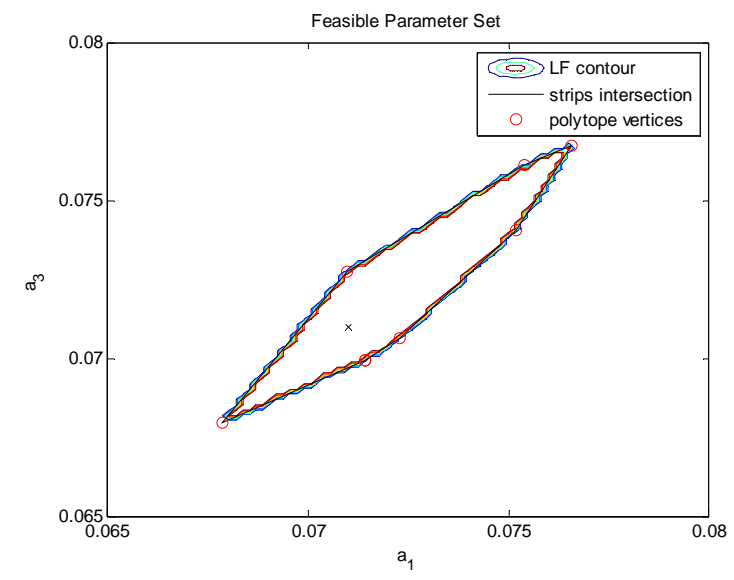

Figure 5. FPS obtained by the likelihood technique

\subsubsection{Nonlinear case}

In the observer case, since the resulting recursive structure is nonlinear in the parameters the strips intersection set-membership technique cannot be applied. By contrast, in the Bayesian approach, the same methodology can be used for either linear or nonlinear systems.

Figure 6 shows the FPS region obtained for the case when an observer with gain $L=0.1$ is used. As expected, the use of the observer leads to a tightened FPS region.

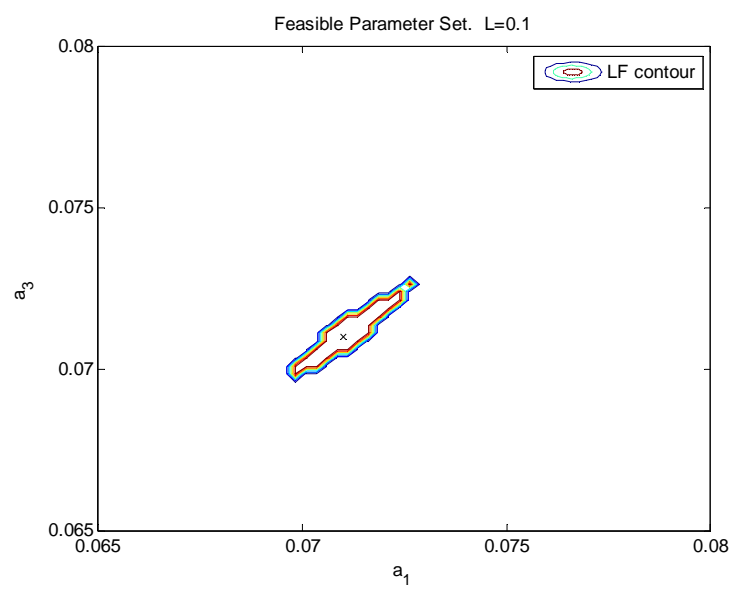

Figure 6. FPS obtained by the likelihood technique in the nonlinear case 


\subsection{Uncertainty estimation in a fault-free scenario for the MIMO case}

Now we consider the MIMO (Multiple Input Multiple Output) case. A set of 21000 measurement data have been obtained for the whole system. Figure 7 shows the steady state final 14000 samples for each tank level. The first $M=500$ samples of this record will be used for calibration purposes.
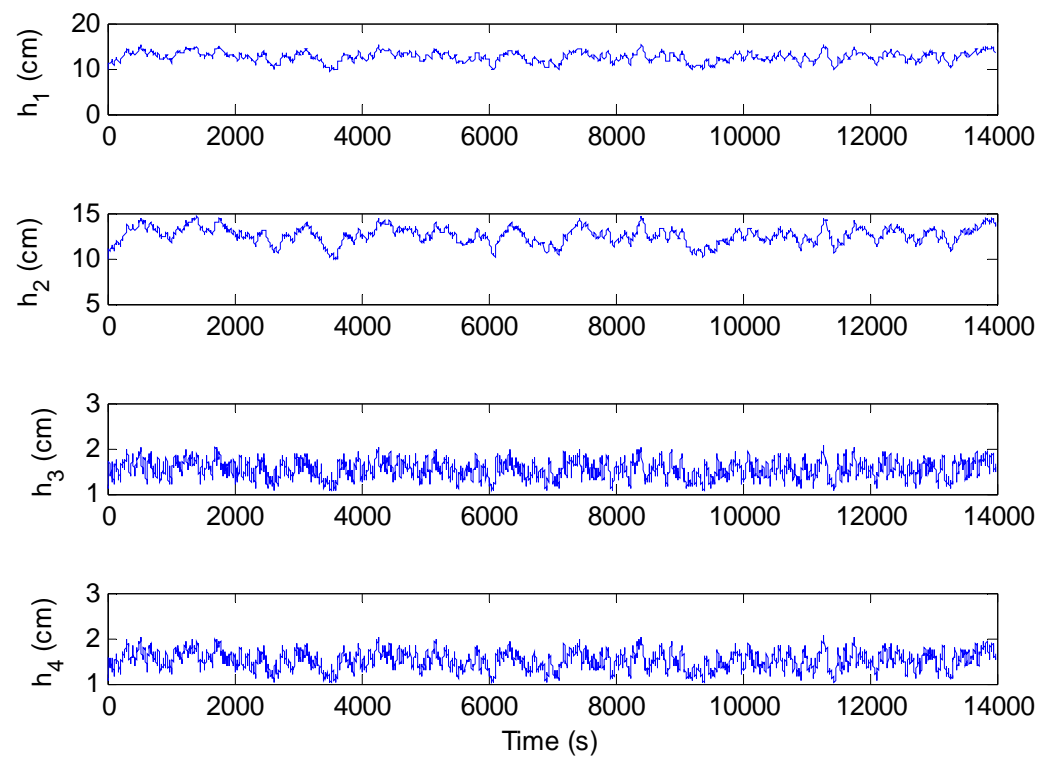

Figure 7. Measurement data for the MIMO case

The system in (26) can be viewed as two independent MIMO systems. In the first one, the inputs are $v_{1}$ and $v_{2}$, and the outputs are $h_{1}$ and $h_{3}$. The uncertain parameters are, again, $a_{1}$ and $a_{3}$.

$$
\begin{aligned}
& \frac{d h_{1}}{d t}=-\frac{a_{1}}{A_{1}} \sqrt{2 g h_{1}}+\frac{a_{3}}{A_{1}} \sqrt{2 g h_{3}}+\frac{\gamma_{1} k_{1}}{A_{1}} v_{1} \\
& \frac{d h_{3}}{d t}=-\frac{a_{3}}{A_{3}} \sqrt{2 g h_{3}}+\frac{\left(1-\gamma_{2}\right) k_{2}}{A_{3}} v_{2}
\end{aligned}
$$

In the second one, the inputs are $v_{1}$ and $v_{2}$, and the outputs are $h_{2}$ and $h_{4}$. The uncertain 
parameters are $a_{2}$ and $a_{4}$.

$$
\begin{aligned}
& \frac{d h_{2}}{d t}=-\frac{a_{2}}{A_{2}} \sqrt{2 g h_{2}}+\frac{a_{4}}{A_{2}} \sqrt{2 g h_{4}}+\frac{\gamma_{2} k_{2}}{A_{2}} v_{2} \\
& \frac{d h_{4}}{d t}=-\frac{a_{4}}{A_{4}} \sqrt{2 g h_{4}}+\frac{\left(1-\gamma_{1}\right) k_{1}}{A_{4}} v_{1}
\end{aligned}
$$

The identified error bounds are $\sigma_{1}=0.1134, \sigma_{2}=0.1098, \sigma_{3}=0.1036$, and $\sigma_{4}=0.1024$

\subsubsection{Set-membership approach}

Firstly, we obtain the uncertainty region for the parameters $a_{1}$ and $a_{3}$ by considering the equation (29) for $h_{1}$ (see Figure 8(a)) and then the uncertainty region for the parameters $a_{1}$ and $a_{3}$ by considering the equation (30) for $h_{3}$ (see Figure 8(b)).

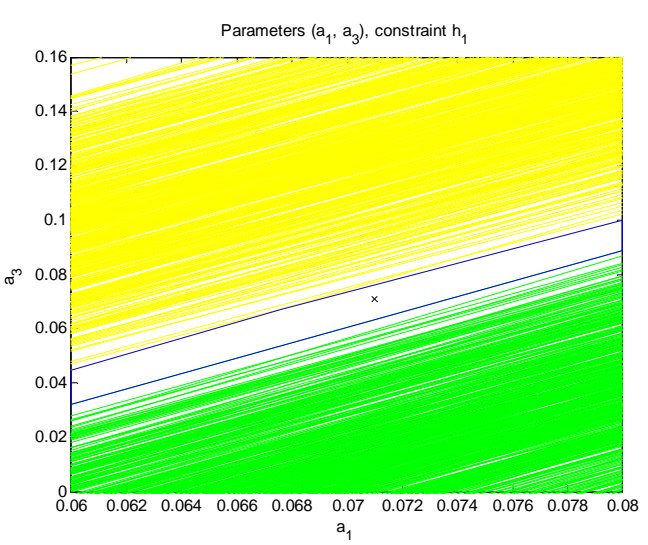

(a)

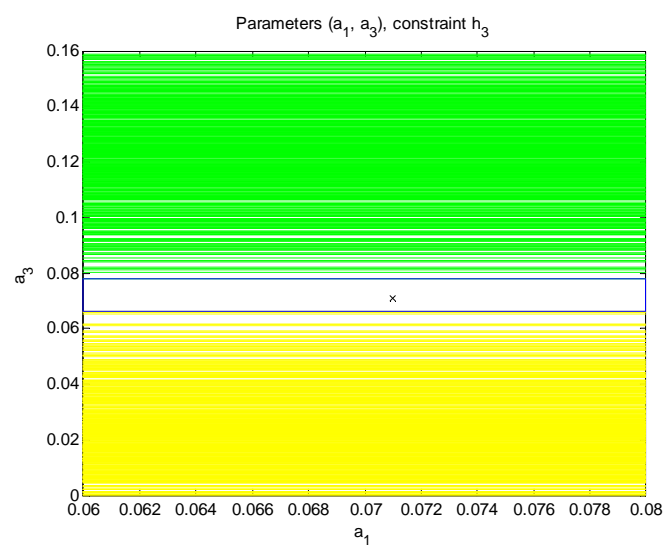

(b)

Figure 8. MIMO case. Uncertainty region for $a_{1}$ and $a_{3}$ considering constraints (a) $h_{1}$

$$
\text { and (b) } h_{3}
$$

The combination of the previous regions leads to the uncertainty region shown in Figure 9(a). Figure 9(b) shows the resulting region for the parameters $a_{2}$ and $a_{4}$ and equations (31) and (32) for $h_{2}$ and $h_{4}$, respectively. 


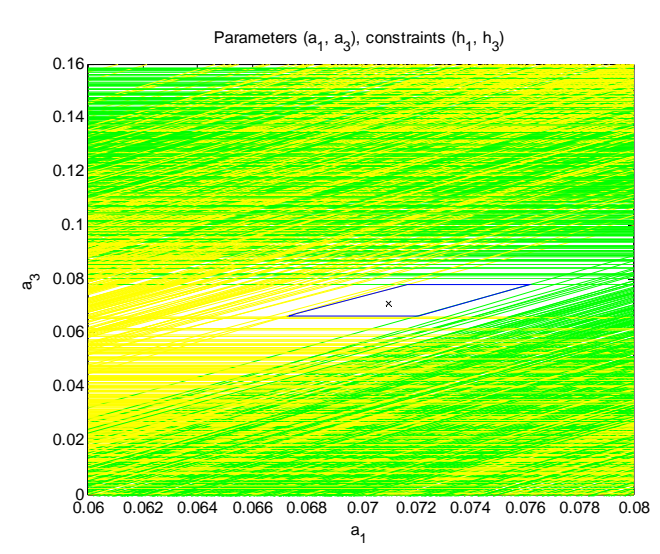

(a)

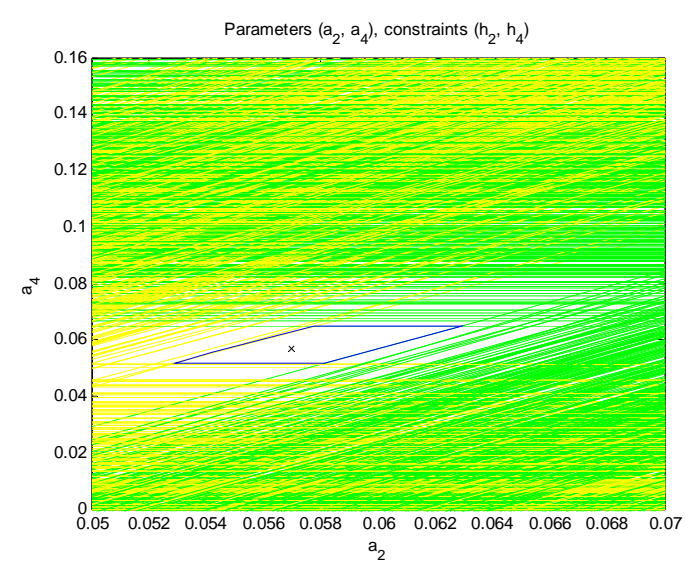

(b)

Figure 9. MIMO case: (a) Final uncertainty region for $a_{1}$ and $a_{3}$ (b) Final uncertainty region for $a_{2}$ and $a_{2}$

\subsubsection{Likelihood approach}

The same region shown in Figure 9(a) can be obtained by computing the likelihood to obtain the measurements $h_{1}, h_{3}$ for each pair of parameters $a_{1}, a_{3}$,

$$
p\left(h_{1}, h_{3} \mid a_{1}, a_{3}\right)=\prod_{k=1}^{M} p_{1}\left(h_{1}-\tilde{h}_{1} \mid a_{1}, a_{3}\right) p_{3}\left(h_{3}-\tilde{h}_{3} \mid a_{1}, a_{3}\right)
$$

by taking a $30 \times 30$ parameters grid, and considering uniform probability distributions for the residuals, $\left(h_{1}-\tilde{h}_{1} \mid a_{1}, a_{3}\right) \sim U\left(-\sigma_{1}, \sigma_{1}\right)$ and $\left(h_{3}-\tilde{h}_{3} \mid a_{1}, a_{3}\right) \sim U\left(-\sigma_{3}, \sigma_{3}\right)$. Figure 10 shows the results for $M=500$ and a grid of $80 \times 80$ values for $a_{1}, a_{3}$ ranging from 0.06 to 0.08 . 


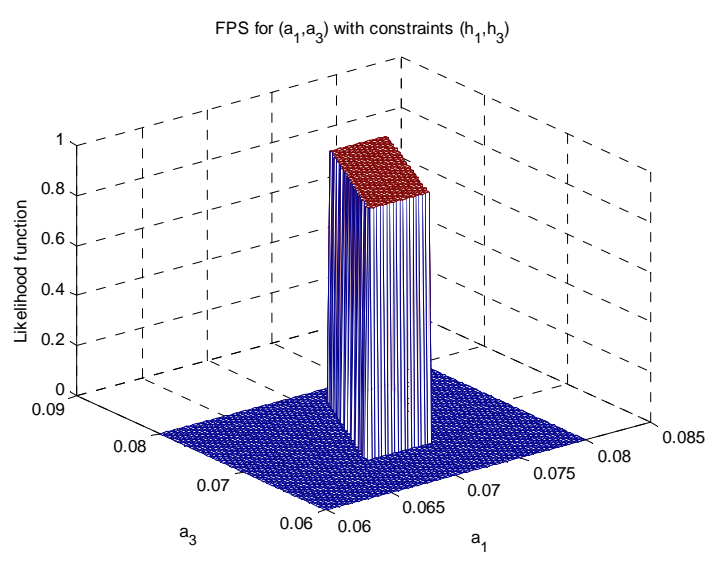

(a)

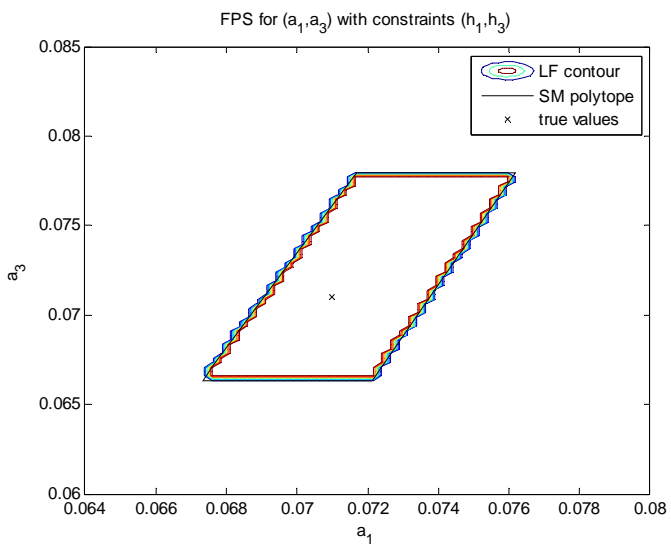

(b)

Figure 10. MIMO case, parameters $a_{1}, a_{3}$. (a) normalized likelihood function, (b) likelihood function contour plot.

Similar results are obtained for each pair of parameters $a_{2}, a_{4}$ by computing the likelihood to obtain the measurements $h_{2}, h_{4}$ (see Figure 11).

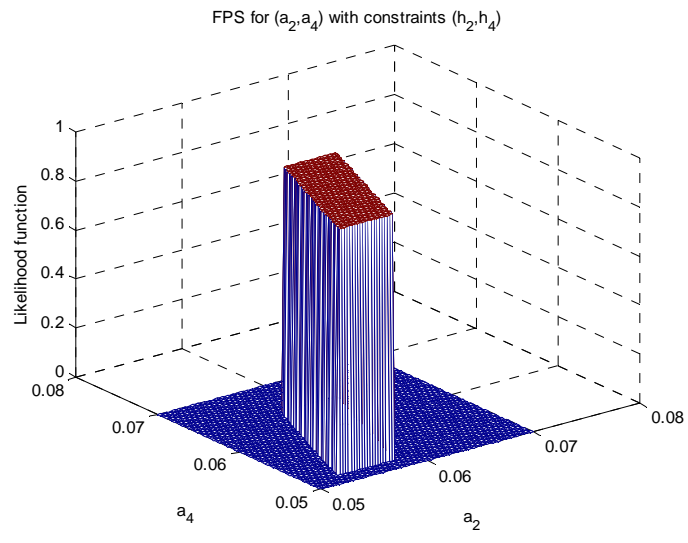

(a)

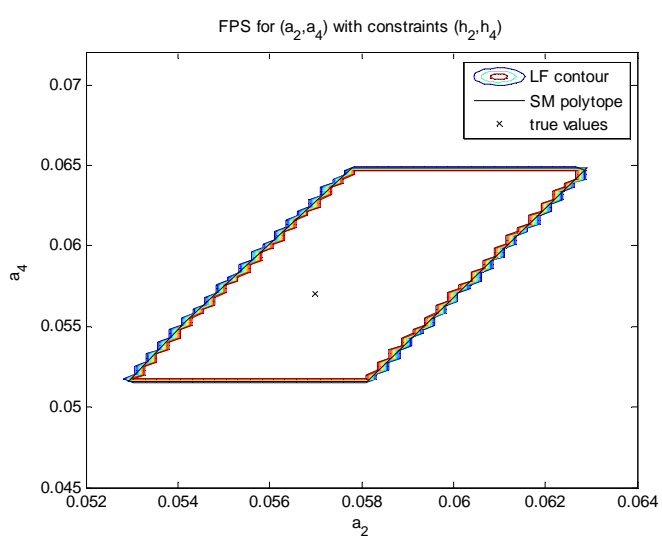

(b)

Figure 11. MIMO case, parameters $a_{2}, a_{4}$. (a) normalized likelihood function, (b) likelihood function contour plot. 


\subsection{Fault detection results}

In order to compare the performance of the strips intersection and likelihood fault detection tests, different fault scenarios have been created by introducing faults when the system is under the operation point shown in Figure 12. For the sake of clearness, in this section we only consider the MISO (Multi Input Single Output) case.

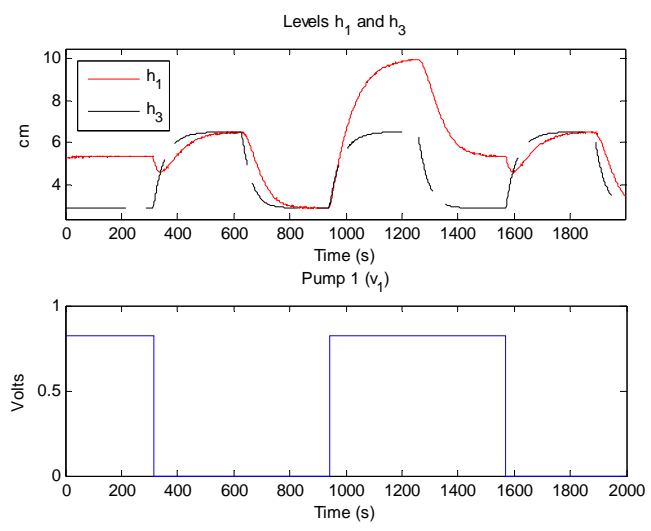

Figure 12. No faulty scenario

Here we illustrate the case when a fault consisting of an additive constant of value 0.035 acting over the parameter $a_{1}$ is introduced at the sample 1201. The faulty behavior is shown in Figure 13.

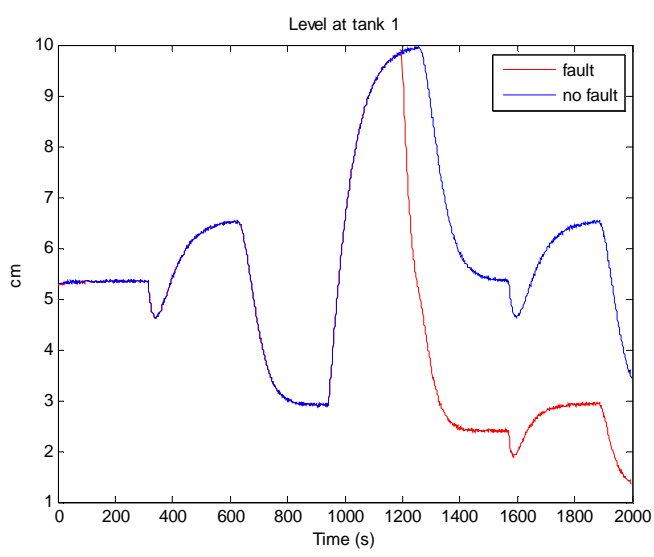

Figure 13. Faulty scenario

Figures 14 and 15 illustrate the fault detection test for the set-membership technique 
based on strip intersection in the linear case.

Figure 14 shows the FPS and the consistency parameter strip $S_{k}$ corresponding to the measured data at $k=1200$. Since the intersection between the FPS and $S_{k}$ is not empty we conclude that the observed deviation from the nominal behavior is due to the model uncertainty and not to a fault. In other words, we say that the measurement at $k=1200$ is consistent with the model and consequently we (correctly) decide that there is no fault.

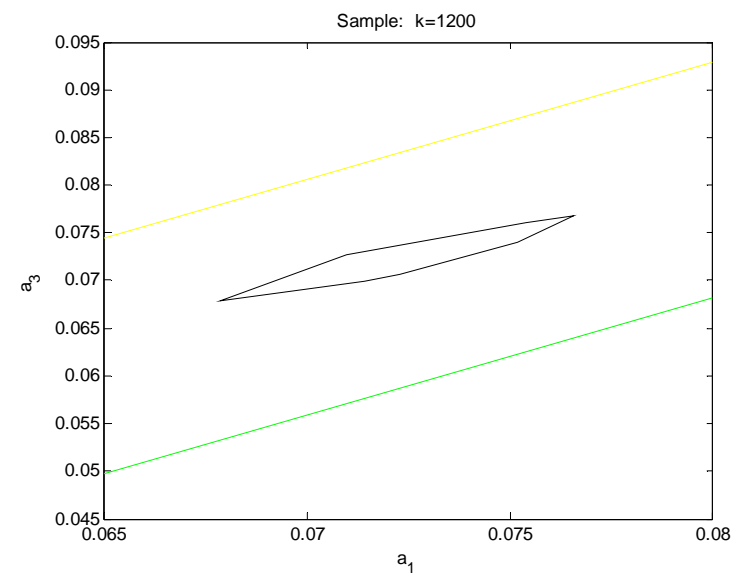

Figure 14. Strips fault detection test in the no faulty scenario

On the other hand, Figure 15 shows that the sample $k=1201$ the FPS and the strip $\mathrm{S}_{k}$ are disjoint, so their intersection is empty. This indicates that the deviation of the behavior cannot be explained by the model uncertainty and therefore we (correctly, again) decide that we have a fault.

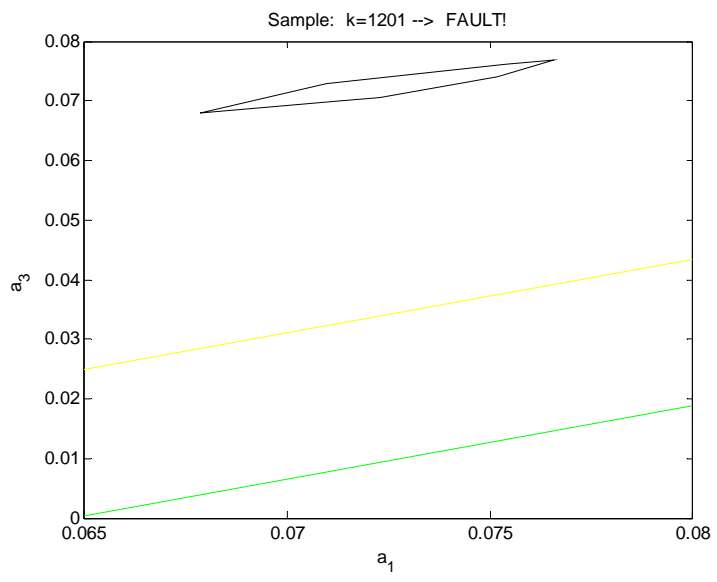


Figure 15. Strips fault detection test when the fault occurs

Finally, Figure 16 and Figure 17 illustrate the likelihood fault detection test in the linear case for a grid of $60 \times 60$ parameters.

Figure 16 shows the initial likelihood function corresponding to the FPS and the likelihood function computed for the new measurement at $k=1200$. The top value in both functions has been scaled to 5 and 10 respectively for comparison purposes. In this sample, the new likelihood totally covers the FPS and so their product is nonzero over the entire FPS region. Since the product of the two likelihood functions is nonzero in at least one point of the grid, we conclude that the data are consistent with the model and therefore we (correctly) decide that there is no fault.

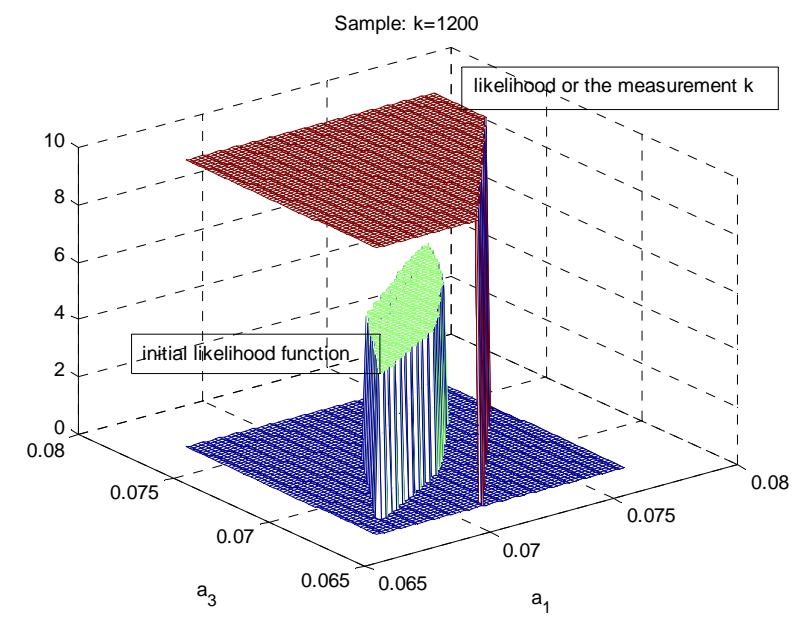

Figure 16. Likelihood fault detection test in the no faulty scenario

On the other hand, Figure 17 illustrates that for the sample $k=1201$ the two likelihood functions are totally separated. This way, their product is zero for all the values over the parameter grid. The conclusion is that the observed deviation of the behavior is not due to the uncertainty because the FPS does not contain any value consistent with the observed data. In this case we (correctly, again) decide that a fault has taken place. 


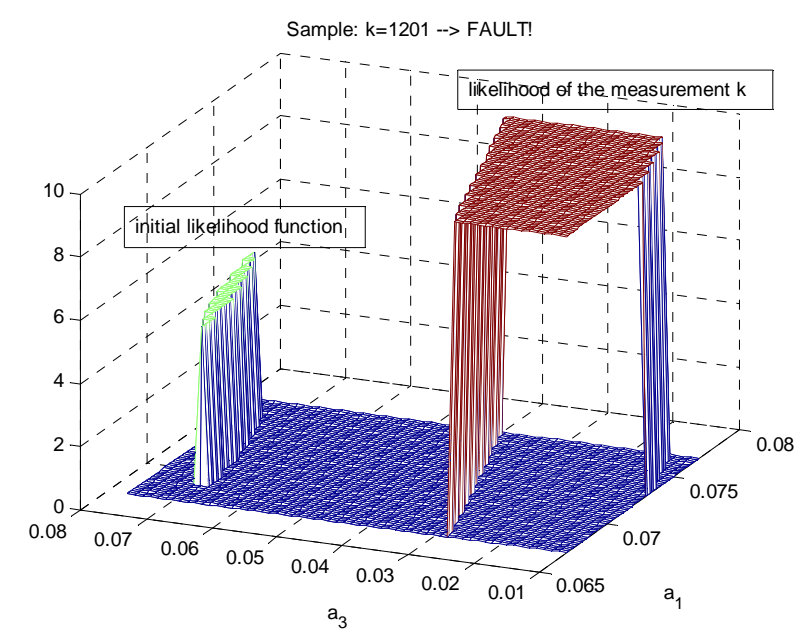

Figure 17. Likelihood fault detection test when the fault occurs

In the example above, for the linear case, the strips intersection test and the likelihood test have obtained the same successful results since the FPS regions were the same.

In the nonlinear case, the comparison cannot be performed since the strips technique cannot deal with structures nonlinear in the parameters. However, for the case of plant plus observer, the likelihood fault detection test has been applied and has successfully detected the fault at the sample 1200. Even more, in the case when an output observer is used, since the resulting FPS regions may be smaller, the methodology is able to detect faults of smaller magnitude. In this example, the likelihood test can detect faults as small as $0.001 \mathrm{~cm}^{2}$, for an observer gain of 0.1 and a $60 \times 60$ parameters grid in the range $\left[\begin{array}{lll}0.076 & 0.066] \times[0.076 & 0.066]\end{array}\right.$.

Once the fault has been detected by Algorithm 2, a new FPS that is consistent with the faulty data and that contains the new parameters of the system can been computed using Algorithm 1 as depicted in the Figure 18. 

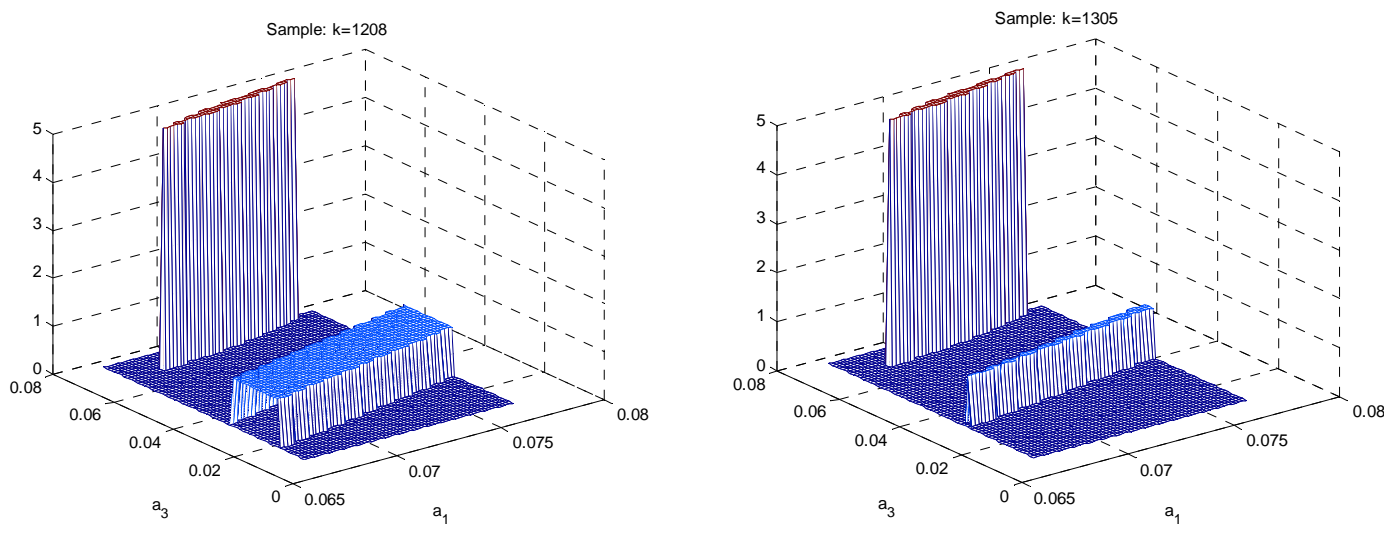

Figure 18. Fault estimation

\section{Conclusion}

In this paper we have presented a new set-membership approach to obtain hard-bounded feasible parameter regions and to perform fault detection on the basis of them. The method is based on a Bayesian framework for system identification assuming that the error bounds are uniform distributed and that the model prior distribution is uniform. In the linear case, the method presented here leads to a point-wise inner approximation of the FPS regions obtained by the strips intersection set-membership technique.

The Bayesian approach presents some advantages and drawbacks compared to the existing deterministic set-membership techniques. Compared to the set-based setmembership technique, although the computation times are similar, the Bayesian technique does not enjoy the guarantee property for the obtained region but in contrast it can deal with nonlinear parameterizations of the system. This is especially interesting when nonlinear structures, such as observers, are used to improve the model estimation. Moreover, the set-membership Bayesian method would allow considering different different noise distributions further than the uniform one assumed by default by the setbased set-membership methods. This would be interesting when the aim is to perform fault detection since less conservative results could be achieved. 
Compared to stochastic approaches such as the particle filtering methods, it is not necessary to obtain samples and weights to estimate the posterior distribution. Given a user defined grid of samples the aim is to see if the associated weight is zero or not. Thus, the presented method does not present the degeneracy problem typical of particle filtering methods. On the other hand, by assuming a uniform prior we are forcing the likelihood dominance, i.e. we are letting the data speak by themselves. If we had reliable prior information about the model parameters, this information could be included in the prior and the resulting region would be tighter.

Although in the quadruple tank case study considered here we have obtained a deterministic region as a particular case of the Bayesian methodology, it has to be stressed that the Bayesian approach is a probabilistic approach, and that this stochastic nature is an advantage rather than the reverse. In a general case, the adequate selection of the model prior probability distributions may lead to probabilistic uncertainty regions that are tighter than the ones obtained by conventional system identification methods.

Regarding the fault detection stage, we have illustrated the detection of faults for the linear case. Since the FPS regions obtained in the calibration stage were the same for the set-membership technique and the Bayesian technique, the two fault detection procedures (strips and likelihood) lead to the same results. In this stage, the Bayesian method presents a computation cost similar to the set-membership strips approach and it can also be implemented on-line.

Finally, it is important to mention that the characterization of the FPS region by means of a point-wise gridding of the initial parameter set presents some shortcomings in the fault detection stage. For example, very small FPS could lie in the spaces between the points of the grid, thus giving zero likelihood for all the points and deciding erroneously 
that a fault has taken place. This drawback can be overcome by taking a denser grid, by implementing an adaptive mechanism in the points' selection stage, or even by generalizing the method in order to characterize the FPS by means model intervals instead of model points. This will be investigated as future research.

\section{Acknowledgment}

This work has been partially grant-funded by CICYT SHERECS DPI-2011-26243 and CICYT WATMAN DPI-2009-13744 of the Spanish Ministry of Education and by i-Sense grant FP7ICT-2009-6-270428 of the European Commission.

\section{References}

Alamo, T., Bravo, J., and Camacho, E. (2005), "Guaranteed state estimation by zonotopes", Automatica, 41(6), pp. 1035-1043.

Arulampalam, M.S., Maskell, S., Gordon, N., and Clapp, T. (2002). "A tutorial on particle filters for online nonlinear/non-Gaussian Bayesian tracking", IEEE Transactions on Signal Processing , 50(2), pp. 174-188.

Blesa, J., Puig, V., and Saludes J. (2012). "Robust fault detection using polytope-based set-membership consistency test”, IET Control Theory \& Applications, 6(12), pp. 1767-1777.

Blesa, J., Puig, V., and Saludes, J. (2011), "Identification for passive robust fault detection using zonotope-based set-membership approaches", International Journal of Adaptive Control and Signal Processing, November 2011, 25(9), pp. 788-812.

Bolstad, W.M. (2010), Understanding Computational Bayesian Statistics, John Wiley.

Campi, M.C. Calafiore, G., Garatti, S. (2009). Interval predictor models: Identification and reliability. Automatica, Volume 45, Issue 8. pp. 382-392.

Chen, J. and Patton R. (1999), Robust Model-Based Fault Diagnosis for Dynamic Systems, Kluwer Academic Publishers.

Eykhoff, P. (1974), System Identification. Parameter and State Estimation, John Wiley. 
Fernandez-Canti, R.M., Tornil-Sin, S., Blesa, J., Puig, V. Nonlinear set-membership identification and fault detection using a Bayesian framework: Application to the wind turbine benchmark. IEEE 52nd Annual Conference on Decision and Control (CDC), 10-13 Decembrer, 2013, Florence, Italy.

Garulli, A., and Reinelt W. (2000), "On model error modelling in set membership identification", Proc. of the SYSID.

Goodwin, G.C., Braslavsky, J.H., and Seron, M.M. (2002), "Non-stationary stochastic embedding for transfer function estimation", Automatica, 38, pp. 47-62.

Ingimundarson, A., V. Puig, T. Álamo, J.M Bravo and P. Guerra. (2008) Robust fault detection using zonotope-based set-membership consistency test. Journal of Adaptive Control and Signal Processing, 23(4): 311-330.

Jaulin, L. (2010), "Probabilistic set-membership approach for robust regression", Journal of Statistical Theory and Practice, 4(1).

Jaulin, L., Kieffer, M., Didrit O., and Walter, E. (2001), “Applied Interval Analysis with Examples in Parameter and State Estimation", Robust Control and Robotics, Springer-Verlag.

Johansson, K.H. (2000), “The Quadruple-Tank Process: A Multivariable Laboratory Process with an Adjustable Zero", IEEE Transactions on Control Systems Technology, 8(3).

Lagoa, C.M., Li, X., and Sznaier M. (2005), "Probabilistically constrained linear programs and risk-adjusted controller design”, SIAM J. Optim., 15(3), pp. 938951.

Milanese, M. and Taragna, M. (2002), “Optimality, approximation, and complexity in set membership $\mathcal{H}_{\infty}$ identification”, IEEE Transactions on Automatic Control, 47(10), pp. 1682-1690.

Milanese, M. and Taragna, M. (2005), “ $\mathcal{H}_{\infty}$ set membership identification: a survey”, Automatica, 41(12), pp. 2019-2032.

Milanese, M., Norton, J.P., Piet-Lahanier, H., and Walter, E. (1996), editors, Bounding approaches to System Identification, Plenum Press, New York, USA.

Ninness, B. and Henriksen, S. (2010), "Bayesian system identification via Markov chain Monte Carlo techniques”, Automatica, 46, pp. 40-51. 
Ninness, B. and Goodwin, G.C. (1995). "Rapprochement between bounded-error and stochastic estimation theory". International Journal of Adaptive Control and Signal Processing.

Peterka, V. (1981), “Bayesian system identification,” Automatica, 17, pp.41-53.

Reinelt, W., A. Garulli, and Ljung, L. (2002), “Comparing different approaches to model error modelling in robust identification", Automatica, 38.

Reppa, V. and Tzes, A. (2011), "Fault detection and diagnosis based on parameter set estimation", IET Control Theory Appl, 5, pp. 69-83.

Robert, C.P. (2001), The Bayesian Choice. $2^{\text {nd }}$ ed., Springer Texts in Statistics. Springer Verlag.

Sánchez Peña, R.S. and Sznaier M. (1998), Robust Systems Theory and Applications, John Wiley \& Sons, Inc.

Schön, T.B., Wills A., and Ninness, B. (2011), "System identification of nonlinear statespace models", Automatica, 47, pp. 39-49.

Sorenson, H.W. (1970), "Least-squares estimation: from Gauss to Kalman", IEEE Spectrum, pp. 63-68.

Verma, V., Gordon, G., Simmons, R., and Thrun, S. (2004), "Real-time fault diagnosis", IEEE Robotics and Automation Magazine, 11(2), pp. 56-66.

Ziegler, G.M. (1995), Lectures on polytopes. Graduate texts in mathematics 152, Springer-Verlag, New York, USA. 